\title{
APPLICATION OF A SIMPLE GPC WITH ROBUST BEHAVIOUR TO A SUGAR FACTORY
}

\author{
Carlos Bordons and Eduardo F. Camacho \\ Dpto. Ingeniería de Sistemns y Automática, Universidad de \\ Sevilla, Spain \\ e-mails: \{bordons, eduardo\}@cartuja.us.es
}

\begin{abstract}
This paper presents the application of a Generalized Predictive Controller (GPC) to the diffusion process in a sugar factory. The controller was embedded in the existing control system, needing the same computational requirements as a PID routine. The control law is extremely simple to compute and the tuning is straightforward since a low order model is used. Due to model uncertainties that appear when working at different operating points; the original GPC algorithm is improved by the use of the so-called $\mathrm{T}$ polynomial, which increases the stability robustness by filtering the predictions. Copyright $(1999$ IFAC:
\end{abstract}

Keywords: Predictive control, Generalized predictive control, Robustness, Process control, Food processing.

\section{INTRODLCTION}

This paper shows an application of a GPC to a process in a sugar refinery. The implementation was carricd out by the authors in collaboration with the firm PROCISA. The refinery is located in Peñafiel (Valladolid, Spain) and belongs to Ebro Agricolas. The controller runs in a ORSI Integral Cube Control System, where the GPC has been included as a library routine which can be incorporated in a control system as easily as the built-in PID routine.

There are many applications of predictive control successfully in use at the present time (Qin and Badgwell, 1997), not only in the process industry but also applications to the control of a diversity of processes (Linkers and Mahfonf, 1994), (Richalet, 1993), (Richalet et al., 1978). MPC is particularly attractive to staff with only a limited knowledge of control, because the concepts are very intuitive, and it can be used to control a grcat variety of processes, from those with relatively simple dynamics to other more complex ones.
The GPC method proposed by Clarke et at. (Clarke et al., 1987a) is a reasonable representative of this family and has become one of the most popular MPC methods, being successfully implemented in many industrial applications (Clarke, 1988). As is well known, the basic idea of GPC is to calculate a scquence of future control signals in such a way that it minimizes a multistage cost function defined over a prediction horizon. The index to be optimized is the expectation of a quadratic function measuring the distance between the predicted system output and some predicted reference sequence over the horizon plus a quadratic function measuring the control effort Generalized Predictive Control provides an explicit solution (in the absence of constraints), it. can cleal with unstable and non-minimum phase plants and incorporates the concept of control horizon as well as the consideration of weighting of control increments in the cost function. The general sct of choices available for GPC leads to a greater variety of control objectives compared to other approaches, some of which can be considered as subsets or limiting cases of GPC. 
A Generalized Predictive Controller results in a linear control law which is easy to implement once the controller parameters are known. The cierivation of the GPC parameters requires, however. some mathematical complexities, which are difficult to solve in some industrial controllers. The industrial application of GPC in small control systems in industry has some difficulties that must be overcome. Apart from needing low computational requirements, it must be accepted by the plant operators. First, the tuning procedure must be simple enough, so that a GPC can be tuned as asily as a PID, and second, the controller must be roluust, that is, it must behave well in the presence of the incvitable modelling errors.

The application shown here combines the power of predictive control with the simplicity and easc of use of the traditional controllers commonly found in industry. In order to improve the robinstness of the closed loop system, the $T$ polynomial has been added to the formulation.

The paper is organised as follows: Section 2 describes the aplication: the diffusion process in a sigar factory. The adaptation of the standard GPC algorithm to a wide class of industrial processes in order to reduce calculations and improve robustmess is presented in section 3 ; section 4 is reclicated to the obtention of the plant model and section 5 to the presentation of operating results. Finally the conclusions of the work are presented in section 5 .

\section{THE DIFUSSION PROCESS}

The factory produces sugar from sugar-beet by means of a serics of processes such as precipitation, cristalization, etc. The process that is controlled in this application is the temperaturc control of the descummed juice in the diffusion.

In order 10 extract the sngar from the beet it is necessary to dilute the saccharose contained in the tuber tissue in water in order to form a juice from which sugar for consumption is obtained.

The juice is obtained in a process known as diffusion. Once the beet has been cut into piccos (called clumks) to increase the interchangable surface, it enters into the macerator (which revolves at a velocity of 1 r.p.m.) where it is mixed with part of the juice coming from the diffusion process (sce figure 1). Part of the juice inside the macerator is recirculated in order to be heated by means of sterm and in this way it maintains the appropriate temperature for maceration. 'The juice from the maceration process passes into the diffusor (a slowly revolving pipe $25 \mathrm{~m}$ long and with a diameter of $6 \mathrm{~m}$ ) where it is mixed with water and all the available sugar content is extracted, leaving

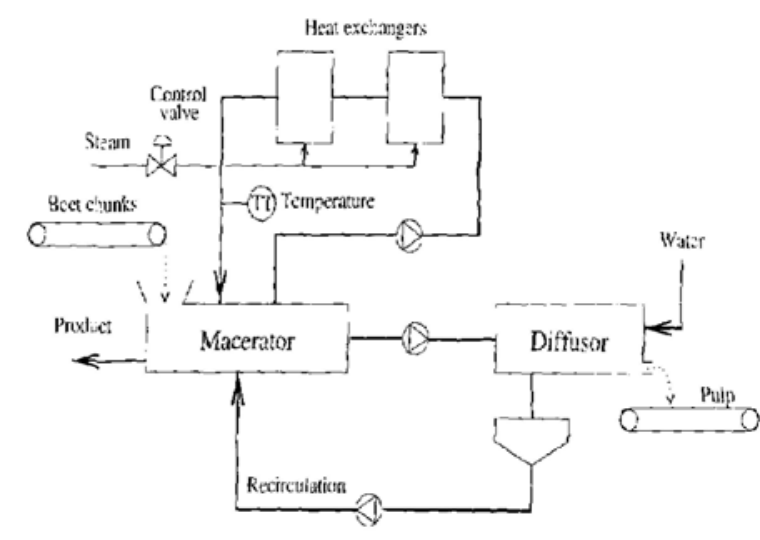

Fig. 1. Diffusion Process in a Sugar Refinery

the pulp as a sub-product. The jujce coming out of the diffusor is recirculated to the macerator, from which the juice already prepared is extracted for the next. process.

In order for the diffusor to work correctly it is necessary to supply thermal energy to the juice during maceration. In order to obtain this objective, part of the juice from the the macerator (about $150 \mathrm{~m}^{3} / \mathrm{h}$ ) is made to recirculate through a battery of exchangers; within these the steam proceeding from the general services of the factory provides the heat needed to obtain optimum maceration. Therefore the controller must adjust the steam valve $(u)$ in order to achicve a determined return temperature to the macerator $(y)$.

The system response is seriously disturbed by changes in the steam pressure, which are frequent. because the steam used in the exchangers has to be shared with other processes which can function in a non-contimusus manner.

\section{PRECOMPLTED GPC}

This paper uses a formulation of Generalized Predictive Control (GPC), easy to implement and tune, that is valid for the majority of industrial processes (Camacho and Bordons, 1995). (Bordons and Camacho, 1998). The method makes use of the fact that a generalized predictive controller results in a control law that can le? described with few parameters. The controller is valid for a wide class of processes in industry and a set of simple functions relating the controller parameters to the process parameters has been obtained. With this set of functions either a fixed or a selftuning GPC can be implemented in a straightforward manner.

Most processes in industry are high order systems that are not suitable for control purposes, but in general it. is possible to approximate the behaviour of such high order processes with a simplified 
model consisting of a first order process combined will a dead time element (Deshpande and Ash, 1981). This type of system is then described by the following transfer function:

$$
G(s)=\frac{K}{1+\tau s} e^{-s^{r} t}
$$

where $K$ is the process static gain, $\tau$ is the time constant or process lag, and $\tau_{d}$ is the dead time or delay. This model is widely used in industry to describe the dynamics of many processes, as shown by the popularity of the reaction curve method and the open loop Ziegler-Nichols PID tuning rules. Obviously better approximations could be oltranied by using higher order models, but this would require identification packages which are not normally available in industry.

When the dead time $\tau_{d i}$ is an integer multiple of the sampling time $T\left(\tau_{d}=d T\right)$, the corresponding discrete transfer function of equation (1) has the form:

$$
G\left(z^{-1}\right)=\frac{b z^{-1}}{1-a z^{-1}} z^{-d}
$$

winere discrete parameters $a, b$ and $d$ can easily be derived from the continuous parameters by discretization of the continuous transfer function, resulting in the following expressions:

$$
a=e^{-\frac{\gamma}{r}} \quad b=K(1-a) \quad d=\frac{\tau_{d}}{T}
$$

Therefore the CARIMA model used for the prediction is :

$$
A\left(z^{-1}\right) y(t)=B\left(z^{-1}\right) u(t-1)+\frac{C\left(z^{-1}\right)}{\Delta} \xi(t)
$$

where $C$ is the noise polynomial. If it is chosen equal to one, the model results:

$$
\left(1-a z^{-1}\right) y(t)=b z^{-1} u(t-d)+\frac{1}{\triangle} \xi(t)
$$

The predictions along the horizon from $t+d+1$ to $t+d+N$ can be calculared by means of the following equation:

$$
\begin{gathered}
\hat{y}(t+d+j \mid t)=(1+a) \hat{y}(t+d+j-1 \mid t)- \\
-a \hat{y}(t+d+j-2 ! t)+b \Delta u(t+j-1)
\end{gathered}
$$

In (Bordons and Camacho, 1998) the GPC algorithm is derived for this kind of processes, leading to the control strategy shown in figure 2 . The phant parameters are used to cormpute the controller coefficients $\left(l_{y 1}, l_{y 2}, l_{r 1}\right)$ as described in (Bordons and Camacho, 1998). These coefficientes are precalculated as a function of the system pole (a) and the control weighting factor $(\lambda)$ with horizons $N_{1}=d+1, N_{2}=d+N, N_{t}=N$,
$N=15$. The values $\hat{y}(t+d j t), \hat{y}(t+d-1 \mid t)$ are obtained by the use of the prediction which basically consists of a model of the plant whicls is projected towards the future with the values of past inputs and outputs and only requires straightforward computation. The control signal is divided by the process static gain in order to get a system with a mitary static gain. The control law is given by:

$$
\begin{aligned}
\Delta u(t)=l_{y 1} \hat{y}(t+d \mid t)+l_{3: 2} \hat{y}(t+d-1 \mid t)+ & \\
& +l_{+1} r(t)
\end{aligned}
$$

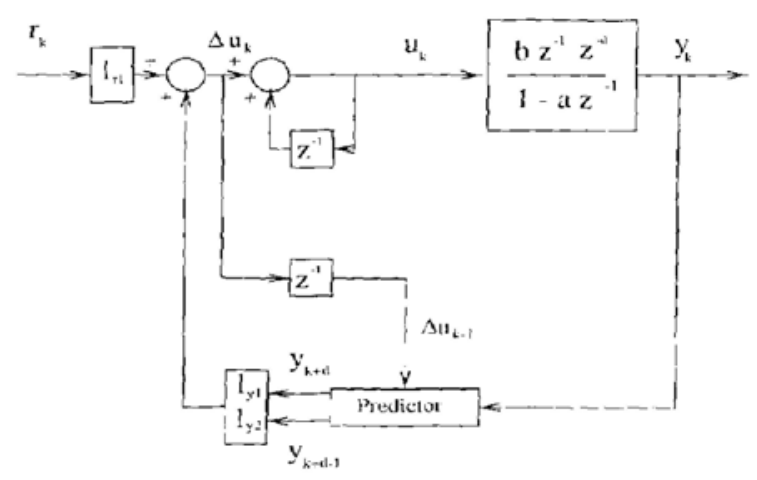

Fig. 2. Control Scheme

The control algorithm reduces to:

1 Compute $k_{3 i}$ as functions of the control weighting factor $\lambda$.

2 Make $l_{3,}=k_{1 i}+k_{2 i} \frac{\hat{a}}{k_{3}-\hat{a}}$ for $i=1,2$ and $l_{r 1}=-l_{y 1}-l_{y 2}$

3 Compute $\hat{y}(t+d \mid t)$ and $\hat{y}(t+d-1 \mid t)$ using equation (5) recursively.

4 Compute control signal $u(t)$ with: $\Delta u(t)=l_{y 1} \hat{y}(t+d j t)+l_{y 2} \hat{y}(t+d-1 j t)$ $+l_{r 1} r(t)$

5 Divide the control signal by the static gain 6 Go to step 2.

It can be seen that the algorithm is really simple and can be easily included in any commercial control system without complex calculation requirements. This algorithm has been succesfully tested in some experimental plants. However, it has also been shown (Camacho and Bordons, 1995) that. altough it is rather robust to gain and time constant uncertainties, it has small robustness to deadtime uncertainties, that are commonly found in real plants. That is why the algorithm must. be modificd to consider this circumstances, since the process to be controlled present that kind of uncertainty (as will be seen later). 
The stability robustness of GPC can be improved witl the use of an observer polynomial, the so('alled $T\left(z^{-1}\right)$ polynomial. In (Clarke and Mohtacli, 1989) a reformulation of the standard GPC afgorithm including this polynomial can be found. In order to do this, the CARIMA model is cxpressed in the form:

$$
A\left(z^{-1}\right) y(t)=B\left(z^{-1}\right) u(t-1)+\frac{T\left(z^{-1}\right)}{\Delta} \xi(t)
$$

Up to now the $T^{\prime}\left(z^{-1}\right)$ has becn considered equal to 1 , describing the most common disturbances $o$ as the colouring polynomial $C\left(z^{-1}\right)$. But it ca11 also be considered as a design parameter. In consequence the predictions will not be optimal but on the other hand robustness in the face of uncertaintics can be achieved, in a similar interpretation as that used by Ljung (Ljung. 1987). Then this polynomial can be considered as a prefilter as well as an observer. The effective use of observers is known to play an essential role in the robust realization of predictive controllers (see (Clarke and Mohtadi, 1989) for the effect of prefiltering on robustness and (Yoon and Clarke, 1994) for guidelines for the selection of $T$ ).

This polynomial can be easily added to the proposed formulation, computing the prediction with the values of inputs and outputs filtered by $T\left(z^{-1}\right)$. Then, the predictor works with $y^{f}(t)=$ $\frac{y(t)}{7\left(\frac{t}{T}\right)}$ and $u^{f}(t)=\frac{u(t)}{\Gamma\left(z^{-1}\right)}$. The actual prediction for the control law is computed as $\hat{y}(t+d)=$ $T^{\prime}\left(z^{-1}\right) \hat{y}^{f}(t+d)$.

The correct choice of the $T$ polynomial is a problem that has not completely been solved, although its effect on the robustness of the closed loop sysiem has been analysed in several papers (Clarke et al., 1987b). (Clarke and Mohtadi, 1989), (Robinson and Clarke, 1991), (Yoon aud Clarke, 1995). In this application, $T$ is made (111a] to) $A\left(z^{-1}\right)\left(1-\beta z^{-1}\right)$, being $\beta$ a value close to the system pole, as suggested in (Yoon and (Iarke, 1995).

\section{PLANT MODEL}

The process is basically a thermal exchange between the stcam and the juice in the pipes of the exchanger, with overdamped behaviour and delay assoriated to the transportation time of the juice throngh pipes about 200 meters long. These consielerations, together with the observation of the development of the system in certain situations. justify the use of a first order model with delay.

A model was identified by its step response. Starting from the conditions of $82.42^{\circ} \mathrm{C}$ and the valve at $57 \%$, the valve was closed to $37 \%$ in order to olsserve the evolution; the new stationary state is obtained at $78.61^{\circ} \mathrm{C}$. The values of gain, time constant and delay can easily be obtained from the response:

$$
\begin{gathered}
K=\frac{82.42-78.61}{57-37}=0.1905 \frac{{ }^{\circ} \mathrm{C}}{\%} \\
\tau=5 \mathrm{~min} \quad \tau_{d}=1 \mathrm{~min} 45 \mathrm{~s}
\end{gathered}
$$

However, it is seen that the system reacts differently when heated to when cooled, the delay being quite a lot greater in the first case. A similar test changing the valve to $57 \%$ again provides values of

$$
K=0.15 \tau=5 \min 20 \mathrm{~s} \quad \tau_{d}=4 \min 50 \mathrm{~s}
$$

Althorgh an adaptive strategy could be useal (with the consequent computational cost), a fixed parameter controller was employed, showing, at the same time, the robustness of the method when using the T-polynomial in presence of modelling errors. The error in the delay, which is the most dangerous, appears in this case. The following values of the model were chosen for this:

$$
K=0.18 \quad \tau=300 \mathrm{~s} \quad \tau_{d}=190 \mathrm{~s}
$$

and sampling time of $T=60 \mathrm{~s}$.

It should be noticed that there are great variations in the delay (that produced on heating is alout threc times greater than that on cooling), due to which it is necessary to introduce the filter $T\left(z^{-1}\right)$ in order to increase the robustness.

\section{OPERATING RESULTS}

With the nominal model chosen, the discrete parameters of the process model are given by:

$$
a=0.8187 \quad b=0.0326 \quad d=3
$$

The controller cocfficients can be computed (sec (Bordons and Camacho, 1998)) calculating $k_{y}(\lambda)$ and then $l_{y 1}, l_{y 2}$ and $l_{r 1}$ as functions of the system poie in the form:

$$
\begin{gathered}
l_{y i}=k_{1 i}+k_{2 i} \frac{a}{k_{3 \eta}-a} \quad i=1,2 \\
l_{r}=-l_{y 1}-l_{y^{2}}
\end{gathered}
$$

$$
\begin{array}{ll}
k_{11}= & -\exp \left(0.3598-0.9127 \lambda+0.3165 \lambda^{2}\right) \\
k_{21}= & -\exp \left(0.0875-1.2309 \lambda+0.5086 \lambda^{2}\right) \\
k_{31}= & 1.05 \\
k_{12}= & \exp (-1.7383-0.40403 \lambda) \\
k_{22}= & \operatorname{cxp}\left(-0.32157-0.81926 \lambda+0.3109 \lambda^{2}\right) \\
k_{32}= & 1.045
\end{array}
$$



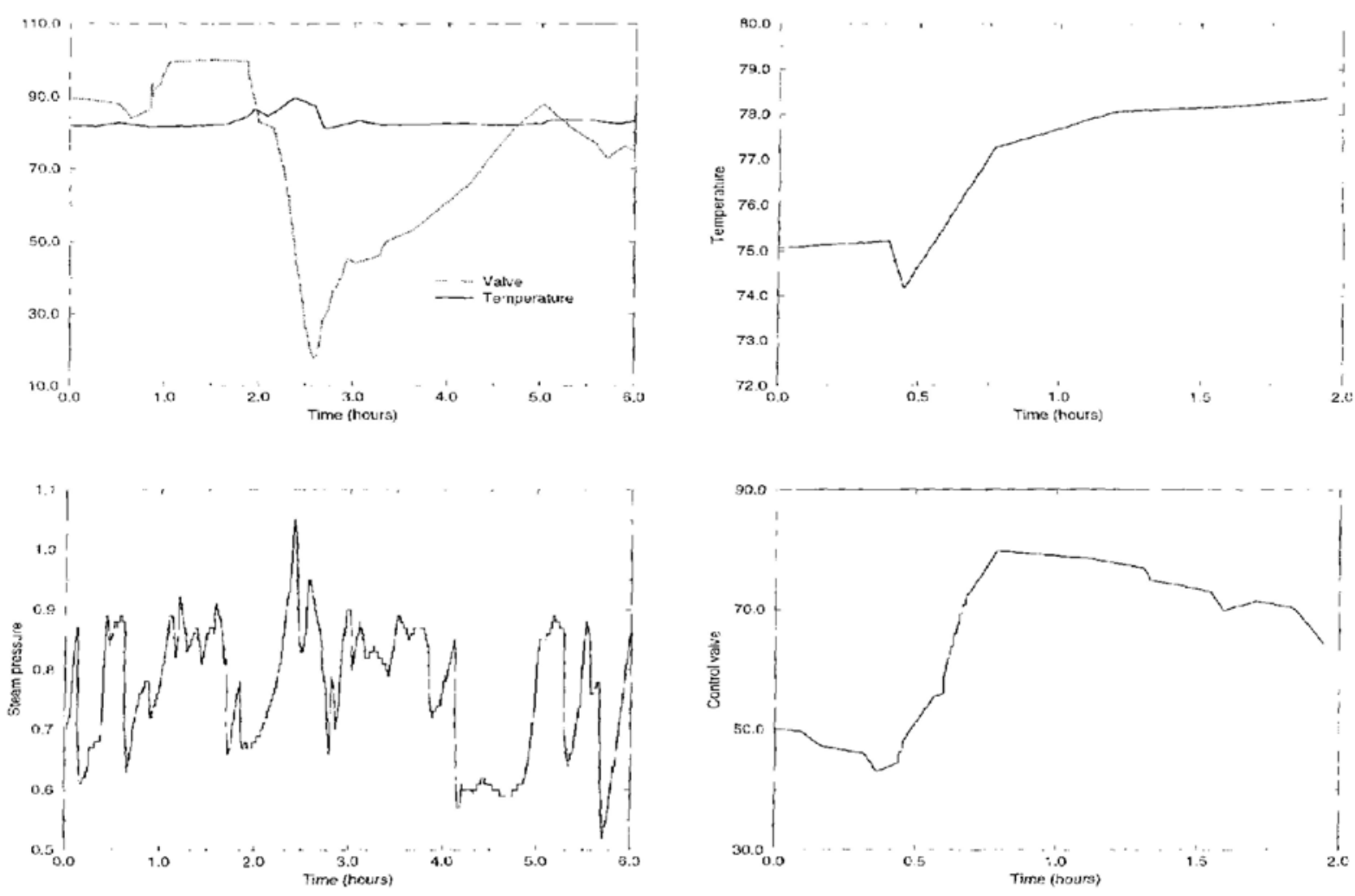

Fig. 3. Systcm response in the presence of external disturbances

As the system pole is in 0.8187 , if a value of $\lambda$ equal to 0.2 is chosen, the controller coefficients are

$$
l_{y 1}=-4.2914 \quad l_{y 2}=2.4165 \quad l_{r 1}=1.8749
$$

The following figures show various moments in operating the temperature control. The behaviour of the controller rejecting the disturbances (brusque variations in the steam pressure and load changes) can be seen in figure 3 . On the othcr hand, figure 4 shows the response to a setpoint change in the jince temperature.

The controller interface allows the process paramctcres to be changed on line. The model is tuned hy the operator as soon as a discrepancy between the actual and the predicted outputs (that appear (s) the screen) is detected.

Following many operational days the operators themselves concluded that a satisfactory model was given by:

$$
\kappa=0.25 \quad \tau=250 \mathrm{~s} \quad \tau_{d}=220 \mathrm{~s}
$$

with a control weighting factor $\lambda=0.1$, a sampling time of 50 seconds and robust filter of $T\left(z^{-1}\right)=A\left(z^{-1}\right)\left(1-0.8 a z^{-1}\right)$, being a the dis(rete pole.

Fig. 4. Setpoint change

It should be emphasised that this controller worked satisfactorily and without interruption until the end of the ycar's campaign, being handled without difficulty by the plant operators. The results obtained using this controller in terms of error variance improved the ones obtained in the last year's campaign, when the process was controlled by a PI.

Notice that these results have not been compared to those of using the controller without the $T$ polynomial. The reason for this is that removing the polynomial made the closed loop unstable (duc to the big dead time uncertainty). As it is a real application, the filter had to be included in the controller when the output started to show a dangerous behaviour.

\section{CONCLUSIONS}

An application of a Generalized Predictive Controller (GPC) to the diffusion process in a sugar factory has been presented. The control law was extremely simple to compute and the tuning was straightforward beacuse of the low order model used. The original GPC algorithm was improved by the use of the $T$ polynomial to increase the stability robustness, since model uncertainties appeared when working at different operating points. Tho 
controller has been succesfully working in the facrory, showing a good behaviour.

\section{ACKNOWLEDGMENTS}

The anthors would like to acknowledge Juan Hermidia from PROCISA by his interest and support in the development of the controller and the perple from Ebro Agricolas by the facilities for testing the controller in the plant. The finantial support of CICYT by projects TAP 96-0884 and TAP 980541 is gratefully appreciated.

\section{REFERENCES}

Bordions, C. and E.F. Camacho (1998). Generalized Predictive Controller for a Wide Class of Industrial Process. IEEE Transaction on Control Systems Technology 6(3), 372-387.

Camacho, E.F. and C. Bordons (1995). Model Predictive Conirol in the Process Iridustry. Springer-Verlag.

Clake, D.W. (1988). Application of Generalized Predictive Control to Industrial Processes. IEEE Control Systems Magazine 122, 49-55.

Clarke, D.W. and C. Mohtadi (1989). Propcrties of Generalized Predictive Control. Autonatica 25(6), 859-875.

Clarke, D.W., C. Mohtadi and P.S. Tuffs (1987a). Generalized Predictive Control. Part I. The Basic: Algorithm. Automatica 23(2), 137-148.

Clarke, D.W., C. Mohtadi and P.S. Tuffs (1087b). Generalized Predictive Control. Part II. Extensions and Interpretations. Automatica 23(2), 149-160.

Deshpande, P.B. and R.H. Ash (1981). Elements of Computer Process Control. ISA.

Linkers; D.A. and M. Mahfonf (1994). Advances in Model-Based Predictive Control. Chap. Gencralized P'redictive Control in Clinical Anaesthesia. Oxford University Press.

Ljung: L. (1987). System Identification. Theory for the user. Prentice-Hall.

Qiil, S.J. and T.A. Badgwell (1997). An Overview of Industrial Model Predictive Control Tecknology. In Chemical Process Control: $\Lambda$ ssessment and New Directions for Research. In: AIChE Synnosium Series 316. 93. Jeffrey $C$. Kantor, Carlos E. Garcia and Brice Carnahan Eds. 93\%-256.

Richalet, J. (1993). Industrial Applications of Model Based Predictive Control. Automatica 29(5), 1251-1274.

Richalet, J., A. Rault, .J.L. Testud and J. Papon (1978). Model Predictive Hewristic: Control: Application to Industrial Processes. Automatica 14(2), 413-428.
Robinson, B.D. and D.W. Clarke (1991). Robustness effects of a prefilter in Generalized Predictive Control. Proceedings IEE, Part D $138,2-8$.

Yoon, T.W. and D.W. Clarke (1994). Advances in Model-Bused Predictive Control. Chap. Towards Robust Adaptive Predictive Control, pp. 402-414. Oxford University Press.

Yoon, T.W. and D.W. Clarke (1995). Observer Design in Receding-Horizon Control. International Journal of Control 2, 151-171. 